\title{
A autoeficácia dos professores para a implementação de práticas inclusivas: contributos para uma reflexão sobre a inclusão educativa ${ }^{1}$
}

Paulo C. Dias ${ }^{a}$

\section{Resumo}

Nos últimos anos, muito mudou em termos de legislação e de práticas para uma escola inclusiva, na preparação das escolas e no desenvolvimento profissional dos docentes. Contudo, é menor a investigação centrada sobre as práticas inclusivas e o sentido de preparação dos professores para uma verdadeira inclusão educativa. Com o presente trabalho pretendemos avaliar a perceção dos professores sobre a sua competência na implementação de práticas inclusivas através da adaptação de uma escala, com 153 docentes do ensino regular e da educação especial. Os resultados permitiram encontrar uma boa estrutura fatorial e fidelidade da escala. Foram encontradas diferenças em função de variáveis pessoais como o género, idade ou habilitações literárias, variáveis profissionais como o tempo de serviço e situação contratual, assim como formação e experiência pessoal com crianças com necessidades especiais. Os resultados são discutidos e as suas implicações para o desenvolvimento profissional docente e para a investigação no âmbito da educação inclusiva.

Palavras-chave: Inclusão. Autoeficácia. Professores. Instrumento.

\section{Introdução}

O movimento inclusivo tem feito um caminho longo e difícil para conseguir dar uma resposta educativa eficaz às crianças e jovens com Necessidades Especiais (NEE). Os desígnios de igualdade, equidade e respeito pela diferença (Ainscow, 1998) têm vindo a ser traduzidos em alterações legislativas e na tentativa de

\footnotetext{
a Universidade Católica Portuguesa - UCP, Centro de Estudos Filosóficos e Humanísticos. Braga, Portugal. Pesquisa financiada ao abrigo do PEsr-OE/FIL/UI0683/2013, da Fundação para a Ciência e Tecnologia (FCT) Ministério da Ciência, Tecnologia e Ensino Superior.
} 
mudança das práticas dos professores num processo longo e, por vezes, doloroso para escolas, professores, pais e alunos.

As mudanças mais marcantes começaram a ser verificadas especialmente a partir dos anos 1980 (Heiman, 2004; Pinto, 2013) com o desenvolvimento social e científico e de políticas educativas promotoras da inclusão. Em Portugal, com a implementação da Lei de Bases do Sistema Educativo, em 1986 (PORTUGAL, 1986), e a entrada em vigor do DL n ${ }^{\circ} 319 / 91$ (PORTUGAL, 1991), implementam-se mudanças organizacionais, nos recursos técnicos e humanos, assiste-se a uma massificação da formação de docentes para dar resposta à entrada das crianças e dos jovens com necessidades especiais nas escolas, embora as respostas continuem muito conotadas com uma perspetiva assistencial (Rodrigues; Nogueira, 2011). Considerando a falta de recursos das escolas e os modelos de formação implementados, tantas vezes sob a forma de programas demasiado curtos para as necessidades destes técnicos, urge equacionar a qualidade das respostas educativas que têm sido implementadas partindo do sentido de eficácia dos professores para a promoção dessas práticas. Mais de 20 anos após a declaração de Salamanca (WORLD CONFERENCE..., 1994), e após a implementação do decreto de lei que regula atualmente a Educação Especial, o DL n ${ }^{\circ} 3 / 2008$ (PORTUGAL, 2008), importa recolher contributos fiáveis para uma avaliação do sentido de preparação dos professores para a implementação destas práticas inclusivas e dos fatores que contribuem para a sua promoção. Na medida em que tivermos ideias claras sobre os avanços e as limitações da formação e das práticas dos professores, atores centrais na promoção destes valores na escola, conseguiremos reunir evidências para a melhoria da formação contínua e das políticas educativas.

Conscientes de que as expectativas sobre a Educação Especial são enormes, seja pelas necessidades dos alunos e das suas famílias, seja pela própria designação "especial" destas respostas, importa avaliar a percepção de eficácia de todos os professores e perceber o seu contributo para a implementação de uma escola inclusiva. Sendo o sentido de eficácia um excelente indicador do comportamento, neste caso, da implementação de práticas inclusivas, começamos por adaptar para o nosso contexto um dos poucos instrumentos que avalia esta dimensão específica: a Escala de Autoeficácia na Implementação de Práticas Inclusivas (Sharma; Loreman; Forlin, 2012). Dessa forma, começamos por apresentar os estudos de adaptação da escala para o contexto português. A partir dos estudos psicométricos deste instrumento, faremos uma exploração dos seus resultados em função de fatores pessoais e profissionais, para perceber eventuais implicações para a formação e políticas educativas inclusivas. 


\section{Autoeficácia dos professores para a implementação de práticas inclusivas ${ }^{2}$}

Em Portugal temos assistido a mudanças profundas na escola, nomeadamente para a promoção de uma cultura inclusiva. Este movimento implica a abertura e o envolvimento aos alunos, independentemente das suas características ou necessidades, o que tem gerado grande reflexão e esforço de adaptação, no sentido de se encontrarem respostas mais eficazes para uma verdadeira inclusão. Curcic (2009), numa comparação internacional sobre a investigação acerca das práticas inclusivas em 18 países, entre os quais Portugal, aponta quatro tópicos emergentes: as preocupações com os resultados dos alunos em contexto inclusivo; a relação entre as crenças e práticas inclusivas dos professores; as filosofias e práticas nas escolas inclusivas; e a relação entre inclusão e exclusão. Apesar dos avanços na legislação, é possível perceber nos vários países e na literatura, a falta de acordo entre investigadores em relação ao que se pode considerar práticas inclusivas e uma distância ainda assinalável em relação à sua verdadeira implementação nas escolas.

Embora diferentes autores utilizem tipologias distintas para descrever a evolução do tipo de acompanhamento, parece consensual o enquadramento da intervenção ao nível da escola, cabendo a esta instituição organizar-se, com os recursos materiais e humanos necessários, para dar resposta às necessidades dos alunos. O mesmo acontece em termos da sua implementação e do papel do professor. Apesar de visões divergentes sobre a melhor forma de concretizar uma escola inclusiva, e da necessidade de organização e orientação da escola, é inegável o papel dos professores para a eficácia das respostas (Greguol; Gobbi; Carraro, 2013). É essencial que os professores se sintam preparados e comprometidos na construção da escola inclusiva, uma vez que lhes cabe um papel central na identificação e acompanhamento dos alunos, mas também na criação de sentimentos de pertença e incentivos à participação e motivação de todos (Correia, 2003, 2005).

Para isso, é essencial a implementação de práticas pedagógicas que estejam intimamente baseadas na evidência (Aguiar et al., 2011). Isto é, o professor deve tentar utilizar a estratégia ou prática pedagógica que melhores resultados tiver demonstrado em situações anteriores empiricamente verificadas junto de alunos com características semelhantes, seja para promover a aprendizagem seja para limitar problemas de comportamento. A partir de estudos de investigação, desenvolvidos com rigor metodológico e avaliação do efeito (Cook; Tankersley; Landrum, 2009), é possível perceber que práticas educativas constituem mais-valia na formação dos

\footnotetext{
2 Trabalho financiado ao abrigo do PEsr-OE/FIL/UI0683/2011, da Fundação para a Ciência e Tecnologia.
} 
educadores e nas respostas aos desafios das práticas educativas. Se isso deveria ser o princípio em todas as intervenções educativas, pelas características da população alvo da Educação Especial, percebe-se a sua particular relevância junto destas crianças e jovens. No entanto, e pese embora estes sejam termos e práticas cada vez mais defendidas e correntes na literatura internacional, são escassos os estudos sobre a sua aplicação no nosso contexto (Aguiar et al., 2011).

Ainda particularmente importante é a colaboração e articulação com os restantes técnicos e famílias, partindo das experiências e dos saberes específicos de todos envolvidos (Roldão, 2007). Esta é tão mais necessária quando consideramos as diferentes tarefas que o acompanhamento eficaz destas crianças e jovens implica, desde a consultoria com os colegas, técnicos, pais e outros profissionais de educação; a realização conjunta das planificações de aulas de diferentes docentes; a elaboração e avaliação dos planos educativos individuais; até ao trabalho com os alunos com NEE e os seus pares para a promoção de atividades de aprendizagem mais ricas e significativas para todos (Correia, 2008; Lima, 2002; Sileo, 2011; Villa; Thousand; Nevin, 2008). Tal é o consenso sobre o seu papel determinante da colaboração no desenvolvimento, aperfeiçoamento e promoção da qualidade dos processos educativos que tem sido salientada por diversos autores (por exemplo, Ainscow, 1997; Ben-Yehuda; Leyser; Last, 2010; Caputo; Langher, 2015; Correia, 2008; Sileo, 2011). Embora este caminho de colaboração possa gerar algumas situações de algum conflito entre os envolvidos (Morgado, 2003; Sileo, 2011), importa a criação de um clima e uma cultura em que todos se possam conhecer, comunicar e partilhar as suas competências e experiências para criar atividades e experiências promotoras da aprendizagem e do desenvolvimento integral (Conderman, 2011; Sileo, 2011; Giangreco; Baumgart; Doyle, 1995).

Considerando estes desafios, importa perceber em que medida os professores sentem competência para os levar a cabo, isto é, em que medida se sentem autoeficazes para a implementação de práticas inclusivas. Para isso, muito contribuíram os trabalhos de Bandura sobre a autoeficácia, que diz respeito aos julgamentos que cada indivíduo faz das suas capacidades para levar a cabo determinada tarefa ou desempenhar determinada actividade e, por isso, é um dos mais fortes preditores do comportamento humano (Bandura, 1997). No fundo, como sublinham Zimmerman e Cleary (2006), a autoeficácia é a crença que cada pessoa tem sobre a sua capacidade de fazer algo, sendo, por isso, determinante para compreender as escolhas que cada pessoa faz, o esforço que despende nas tarefas ou mesmo a sua persistência perante o desafio e a qualidade da sua realização (Bandura, 1997; Schunk; Meece, 2005). Embora tenha inicialmente sido descrita como um traço unidimensional, os estudos tem mostrado que se trata de um construto multidimensional e que se dirige às diferentes atividades 
em que o sujeito se aplica (Bandura, 1997). No contexto do ensino, tem sido estudada em termos de autoeficácia dos professores para a gestão da sala de aula, do investimento que fazem no ensino, dos objetivos e níveis de aspiração dos alunos (Tschannen-Moran; Woolfolk Hoy, 2001; Tschannen-Moran; Woolfolk Hoy; Hoy, 1998). Numa perspetiva de promoção da educação inclusiva, poucos têm sido os estudos realizados neste âmbito. No entanto, contributos recentes, e com base na comparação de países diversos, suportam esta perspetiva multifatorial da autoeficácia para a inclusão (Malinen et al., 2013; Sharma; Loreman; Forlin, 2012). Partindo do modelo tripartido de Tschannen-Moran (Tschannen-Moran; Woolfolk Hoy; Hoy, 1998; Tschannen-Moran; Woolfolk Hoy, 2001), que têm aprofundado o estudo da autoeficácia para os professores, Sharma Loreman e Forlin (2012) adaptam o modelo para um contexto inclusivo, desenvolvendo um instrumento que se verificou ajustado quando aplicado no Canadá, Austrália, Hong Kong e Índia. Esses resultados foram suportados, mais tarde, noutro estudo internacional realizado por Malinen et al. (2013), com a informação que decorre da sua aplicação e avaliação na Finlândia, China e África do Sul. Apesar de diferenças entre os países, os estudos suportam uma conceção da autoeficácia dos professores que se concretiza em três fatores essenciais: eficácia na utilização de práticas pedagógicas inclusivas; eficácia na colaboração com os restantes docentes, técnicos e família; e eficácia a lidar com os comportamentos disruptivos.

Com base nestas informações, o presente trabalho pretende avaliar a perceção dos professores Portugueses sobre a sua competência para ensinar, eficazmente, numa escola inclusiva. Para isso, começaremos por apresentar os dados da tradução e adaptação da Escala de Autoeficácia na Implementação de Práticas Inclusivas (Sharma; Loreman; Forlin, 2012), para, com base na estrutura encontrada, explorar o papel de fatores pessoais e profissionais na autoeficácia para a inclusão.

\section{Metodologia}

\subsection{Amostra}

A amostra deste estudo é constituída por 153 professores de vários agrupamentos do norte do país (Tabela 1). Desse grupo, 27 homens (17,6\%) e 126 mulheres $(82,4 \%)$, com idades entre os 28 e os 58 anos $(\mathrm{M}=42,79, \mathrm{DP}=7,391)$, a maioria licenciados $(\mathrm{n}=119,82,1 \%)$ e professor do quadro $(\mathrm{n}=120,81,1 \%)$ com tempo de serviço superior a 15 anos $(n=97,66,4 \%)$. Foram 77 os que já exerceram o seu serviço como professor de apoio educativo ou na educação especial $(51,0 \%)$, 21 fizeram formação especializada na Educação Especial (13,7\%), 81 participaram em ações de formação contínua na área das $\operatorname{NEE~}(53,6 \%)$, e 36 têm nas suas relações pessoais, amigos ou familiares com NEE $(23,8 \%)$. 
Tabela 1. Descrição da amostra.

\begin{tabular}{lccc}
\hline & Grupo & N & $\%$ \\
\hline \multirow{2}{*}{ Sexo } & Masculino & 27 & 17,6 \\
& Feminino & 126 & 82,4 \\
Idade & Até 29 anos & 8 & 5,3 \\
& 30 a 39 anos & 39 & 25,7 \\
& 40 a 49 anos & 78 & 51,3 \\
Grau académico & 50 ou mais anos & 27 & 17,8 \\
& Licenciatura & 119 & 82,1 \\
Situação profissional & Pós-Graduação & 19 & 13,1 \\
& Mestrado & 7 & 4,8 \\
Exerceu funções na Educação Especial & Professor de quadro & 120 & 81,1 \\
& Professor contratado & 28 & 18,9 \\
Experiência com alunos com NEE & Sim & 77 & 51,0 \\
& Não & 74 & 49,0 \\
\hline
\end{tabular}

Fonte: Elaborado pelo autor (2015).

\subsection{Instrumentos}

Para a recolha de dados foi utilizado um questionário sociodemográfico, construído pelos autores, para a recolha de dados pessoais e profissionais, nomeadamente idade, sexo, habilitações académicas, anos de serviço, experiência com alunos de NEE, experiência docente na Educação Regular ou Especial.

Foi ainda utilizada a Escala de Autoeficácia na Implementação de Práticas Inclusivas (Sharma; Loreman; Forlin, 2012), composta por 18 itens com uma escala de resposta fechada, desde 1 (discordo totalmente) a 6 (concordo totalmente). Os itens avaliam três dimensões: estratégias de ensino inclusivas (seis itens, por exemplo: Consigo utilizar diversas estratégias de avaliação, por exemplo, portfólio, testes adaptados, avaliação com base no desempenho etc.), eficácia na colaboração (quatro itens, por exemplo: Sou capaz de trabalhar conjuntamente com outros profissionais e funcionários [por exemplo, auxiliares, outros professores] no ensino a alunos com NEE, na sala de aula), eficácia no controlo de comportamentos (seis itens, por exemplo: Sinto confiança na minha capacidade para prevenir comportamentos indisciplinados, na sala de aula, antes da sua ocorrência). 


\subsection{Procedimentos}

Para a realização do estudo, foram contactados os autores dos instrumentos, no sentido de solicitar autorização para a adaptação dos instrumentos, e os diretores dos agrupamentos para a permissão para administrar os questionários junto dos professores ${ }^{3}$. O protocolo foi administrado a uma amostra não probabilística, seleccionada por motivos de conveniência, nomeadamente a proximidade geográfica com o centro de investigação. Depois de recolhidos, os dados foram codificados e analisados com o recurso ao programa Statistical Package for Social Sciences (SPSS). Foi utilizada uma análise fatorial exploratória para avaliar a validade da medida, isto é, a partir dos dados, encontrar uma solução teórica e empiricamente sustentável. Para avaliar a fidelidade do instrumento, foi utilizado o alfa de Cronbach. Posteriormente, foram efetuados estudos inferenciais para explorar o papel de variáveis pessoais e profissionais, nomeadamente de experiência inclusiva, no sentido de eficácia dos professores.

\section{Resultados}

\subsection{Estudos de adaptação da medida}

Os 18 itens foram submetidos ao teste de esfericidade de Bartlett e Kaiser-Meyer-Olkin, obtendo-se respetivamente os valores de 1301.247 ( $\mathrm{p} \leq 0,001)$ e 0,825 , o que permitiu perceber a adequadabilidade da base de dados para a análise fatorial. Os itens foram então submetidos à análise fatorial exploratória, pelo método de componentes principais com rotação varimax, no sentido de avaliar a sua proximidade com a estrutura original. Foi possível encontrar uma solução com três fatores distintos com evidências interpretáveis (Tabela 2), que permite explicar 53,521\% da variância total. O primeiro fator, com um valor próprio de 6,795 explica 33,974\% da variância e integra os itens da dimensão relativa à colaboração com os colegas docentes e família dos alunos. $\mathrm{O}$ segundo fator, com valor próprio de 2,036 , que explica $10,180 \%$ da variância, agrupa os seis itens relativos ao sentido de eficácia para a promoção de estratégias pedagógicas inclusivas. E, por fim, com valor próprio de 1,873 , que explica $9,367 \%$ da variância, o terceiro fator agrupa os itens relativos à eficácia para o controlo de comportamentos disruptivos. Apesar de a generalidade dos itens apresentar um valor de saturação superior a 0,40 , o item 9 apresenta um valor mais baixo, que deve ser considerado em futuros estudos.

\footnotetext{
3 Trabalho financiado ao abrigo do PEsr-OE/FIL/UI0683/2011, da Fundação para a Ciência e Tecnologia.
} 
Tabela 2. Análise factorial exploratória da escala.

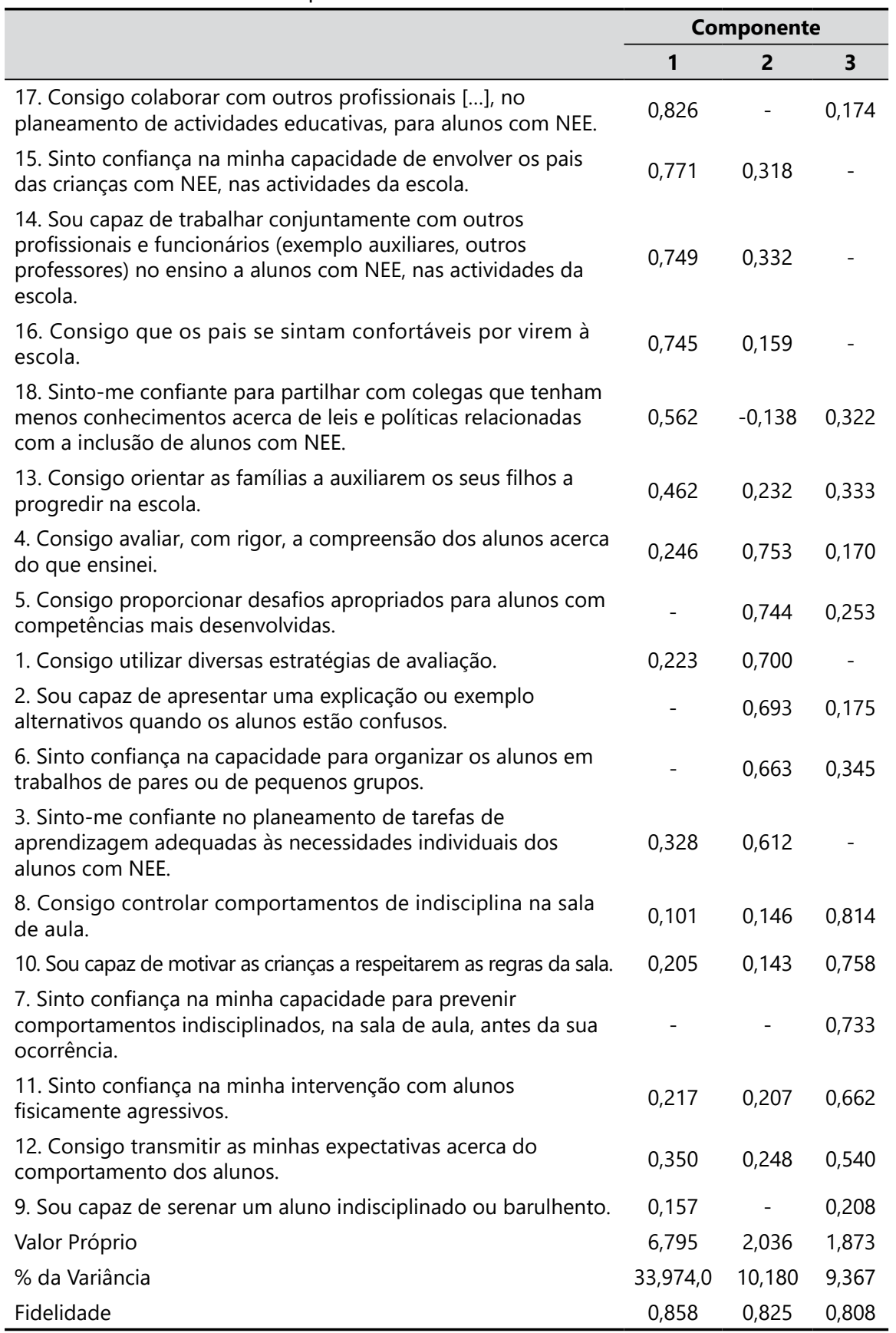

Fonte: Elaborado pelos autores (2015). 
No que diz respeito à consistência interna, o alfa de Cronbach total encontrado foi de 0,897 , variando entre os 0,858 no primeiro fator e 0,808 no terceiro, valores que significam uma boa fidelidade do instrumento.

\subsection{Autoeficácia em função de variáveis pessoais}

Foram posteriormente realizadas análises inferenciais para tentar perceber que fatores podem ter um papel significativo na autoeficácia dos docentes para a implementação de práticas inclusivas. Os resultados permitiram verificar diferenças estatisticamente significativas no total da escala de autoeficácia $\left[\mathrm{t}_{(143)}=2,544, \mathrm{p}=0,012\right]$, assim como nas diferentes subescalas, seja na autoeficácia para a implementação das estratégias inclusivas $\left[\mathrm{t}_{(143)}=2,255, \mathrm{p}=0,026\right]$, na autoeficácia na colaboração [ $\left.\mathrm{t}_{(147)}=2,298, \mathrm{p}=0,023\right]$, como na autoeficácia na gestão de comportamentos indisciplinados $\left[\mathrm{t}_{(149)}=2,413, \mathrm{p}=0,017\right]$. Em todas as dimensões, os homens apresentaram pontuações superiores. Já no que diz respeito à idade, encontrou-se uma relação estatisticamente significativa, negativa e fraca, com a pontuação total da escala $(r=-0,230, p=0,006)$, e nas dimensões relativas à eficácia na utilização de estratégias inclusivas $(\mathrm{r}=-0,190$, $\mathrm{p}=0,022)$ e à autoeficácia na colaboração com outros técnicos ou profissionais $(\mathrm{r}=-0,291, \mathrm{p}<0,01)$.

Analisando os dados em função das habilitações, encontramos diferenças significativas apenas na subescala da colaboração $(F=3,920, p=0,022)$, sendo a média superior encontrada entre docentes com pós-graduação $(\mathrm{M}=21,268, \mathrm{DP}=1,892)$ e a mais baixa entre os docentes com licenciatura $(\mathrm{M}=19,983, \mathrm{DP}=2,126)$.

\subsection{Autoeficácia para as práticas inclusivas em função da experiência profissional e inclusiva}

Quando consideramos a experiência e a situação profissional, foram encontradas diferenças significativas apenas na dimensão relativa à colaboração $\left[\mathrm{t}_{(142)}=-2,099, \mathrm{p}=0,038\right]$, com médias superiores entre os professores contratados. Também no que diz respeito ao tempo de serviço, os dados permitem perceber uma relação estatisticamente significativa e negativa entre o tempo de serviço e a pontuação total da escala $(r=-0,201, p=0,018)$, assim como na colaboração $(\mathrm{r}=-0,256, \mathrm{p}=0,002)$.

Considerando a experiência no exercício de funções na Educação Especial, encontramos diferenças significativas em todas as dimensões $(p<0,05)$, com pontuações médias superiores entre os professores com experiência inclusiva. 
Neste contexto, assinalam-se ainda as diferenças em todas as dimensões em função da formação inicial e contínua na área da Educação Especial, assim como entre os professores que têm pessoas com Necessidades Especiais entre os seus contactos pessoais $(\mathrm{p}<0,01)$ (Tabela 3 ).

Tabela 3. Autoeficácia em função da situação profissional e experiência na Educação Especial.

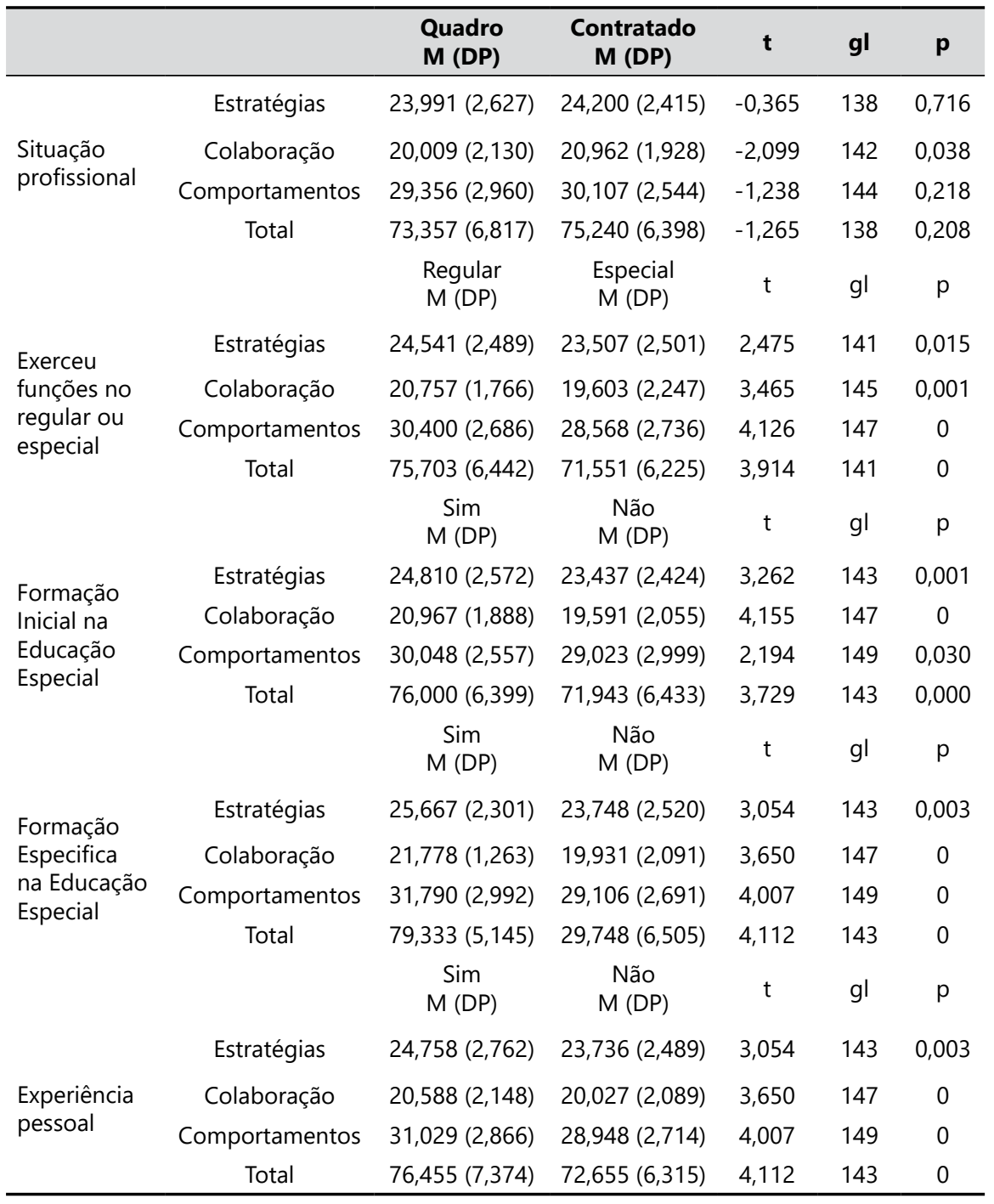

Fonte: Elaborado pelo autor (2015). 


\section{Discussão}

O presente trabalho, eminentemente exploratório, pretendia analisar a percepção de competência dos professores relativamente à inclusão. Num contexto em que se assiste a um conjunto de transformações nas escolas e a uma necessidade de inclusão de alunos com características nas escolas, importa contribuir para adaptação de instrumentos de investigação científica que nos permitam avaliar estas competências para intervir e propor alterações formativas e legislativas com base em evidências.

Assim, começamos por fazer o estudo de adaptação portuguesa da Escala de Autoeficácia na Implementação de Práticas Inclusivas (Sharma; Loreman; Forlin, 2012), um instrumento recente, mas que tem sido alvo de adaptações e estudos multiculturais (Malinen et al., 2013; Sharma; Loreman; Forlin, 2012). Tal como nesses estudos, o nosso trabalho encontrou boas propriedades psicométricas da escala na nossa amostra, o que pode ser verificado pela saturação dos itens nas dimensões previstas, e com valores superiores a 0,4 na sua generalidade. Esses dados de validade são complementados por valores de fidelidade superiores a 0,8 , o que também pode ser considerado muito adequado. Apenas um dos itens, o item 9 (Sou capaz de serenar um aluno indisciplinado ou barulhento) apresenta valores de saturação mais baixos, o que deve ser analisado em próximos estudos, seja com a sua reformulação ou eliminação, para manter a validade e fidelidade da medida. Garante-se, no entanto, resultados muito promissores quanto à utilização deste instrumento em estudos futuros.

Para explorar os fatores que podem explicar diferentes níveis de autoeficácia para as práticas inclusivas, foi testado o papel de algumas variáveis pessoais, profissionais e de experiência inclusiva. As variáveis pessoais parecem menos citadas na literatura, mas seguem, genericamente, no sentido do presente estudo, com pontuações médias mais baixas entre as mulheres (Kiviet; Mji, 2003; Riggs, 1991). Embora seja difícil explicar estas diferenças, elas podem estar intimamente associadas ao género, uma vez que diferenças na autoeficácia para situações de desempenho são encontradas desde muito cedo (Huang, 2013). Também foi possível perceber menor autoeficácia ao longo da idade, o que sugere que os professores mais velhos se sentem menos competentes e, portanto, menos preparados para promover a inclusão. Este é um desafio particularmente emergente quando percebemos a falta de entrada de professores mais novos nas escolas, além da falta de oportunidades ou de estímulos à formação contínua adequada às necessidades dos professores. Coloca-se, portanto, no centro da agenda, a necessidade de formação contínua específica para a preparação dos professores para a inclusão de todos os alunos. 
As funções e a formação específica na educação de crianças com NEE aparecem como fatores diferenciadores na perceção da colaboração e da eficácia. Naturalmente, pela sua formação e exercício profissional, percebe-se que os professores que trabalham na Educação Especial têm maior sentido de eficácia para as práticas inclusivas, o que salienta o papel da formação. Este tem sido um tópico apontado frequentemente na investigação (por exemplo, Ali; Mustapha; Jelas, 2006; Harvey et al., 2010), especialmente porque a educação inclusiva implica estratégias específicas que a tornem verdadeiramente especial (Cook; Schirmer, 2003; Cook, Tankersley; Landrum, 2009), e respondam às necessidades das crianças e famílias. No presente estudo, esse fator aparece sublinhado, sendo superior a autoeficácia dos professores com formação inicial e contínua neste âmbito. Embora diversos estudos apontem para uma atitude genericamente positiva em relação à inclusão, seja dos professores como dos pais (por exemplo, Dias; Leal; Diáz, 2013; Freitas et al., 2015), parecem existir diferenças notáveis na perceção de competência para lidar com este desafio na sala de aula. Pela falta de recursos e de formação adequada para responder a necessidades tão distintas, parece reforçar-se a necessidade de partilha e de procura de respostas colaborativas entre os docentes, para que as dificuldades sejam entendidas e resolvidas por toda comunidade escolar, e não uma preocupação única e exclusiva dos professores especializados da Educação Especial (Morgado, 2003).

Outra variável que aparece como significativa na explicação da perceção de competência para a inclusão é a experiência pessoal com pessoas com Necessidades Especiais. Embora não sejam surpreendentes, estes dados sublinham a importância da proximidade e do conhecimento apropriado para uma atitude e sensação de competência para dar as respostas adequadas. Sendo uma das barreiras a acessibilidade da informação e do conhecimento científico, importa reforçar o investimento em formação específica, com uma forte componente prática, que permita não apenas o acesso a essa informação, mas o desenvolvimento de competências técnicas e pessoais que permitam a sua tradução nas práticas pedagógicas e colaborativas para os profissionais que dela necessitam (Cook et al., 2003).

\section{Conclusões}

Embora tenham passado mais de 20 anos sobre a declaração de Salamanca (WORLD CONFERENCE..., 1994), a investigação tem demonstrado ainda a existência de desafios importantes na sua implementação (Dias et al., 2013), seja no âmbito da formação adequada, nos recursos e na necessidade de perceber a constante necessidade de desenvolvimento profissional para responder às características dos alunos. Com o presente trabalho, parece claro um contributo 
para a investigação e algumas reflexões para a promoção de uma escola inclusiva. Os estudos psicométricos permitiram confirmar a adaptação da escala para a população portuguesa, podendo ser um contributo para os restantes países de Língua Portuguesa. Ainda, o estudo permitiu identificar variáveis que nos permitem perceber o perfil do professor com maior autoeficácia para a implementação de práticas inclusivas.

Apesar dos contributos, é de considerar que este é um primeiro trabalho no nosso contexto, pelo que se sugere a necessidade de replicar esta investigação junto de novas amostras, mais diversificadas e representativas da população portuguesa. Futuros estudos deverão incluir outras variáveis que nos permitam criar modelos mais abrangentes para compreender melhor esta realidade e intervir, no sentido de implementar verdadeiros modelos colaborativos na educação inclusiva. Sugere-se ainda a utilização desta medida para a avaliação do efeito da formação contínua, e de diferentes modelos de desenvolvimento profissional docente, no sentido de promover a melhoria das práticas inclusivas nas escolas.

Do trabalho, resulta ainda um repto para a colaboração entre a academia e as escolas. Nos últimos anos, têm sido evidentes os avanços ao nível da legislação, dos recursos e da investigação centrada na escola inclusiva. Enquanto os investigadores sentem frequentemente a incompreensão das implicações do trabalho, os professores "queixam-se" da falta de aplicabilidade da investigação ao mundo real e fraca disseminação do conhecimento (Spencer; Detrich; Slocum, 2012). Para uma resposta eficaz a este desafio importa, portanto, reforçar as ligações entre as universidades e as instituições educativas para melhorar as competências dos docentes e criar as condições para uma mudança de práticas dos professores e criação de uma cultura inclusiva em contexto escolar (Ainscow, 1998; Correia, 2003, 2005). 


\section{Teachers' self-efficacy toward the implementation of inclusive practices: Contributions for a reflection about school inclusion}

\section{Abstract}

In recent years, much has changed in legislation and practices for an effective inclusive education, in the preparation of schools and in teachers training. However, less research is focused on inclusive practices and teacher sense of efficacy for the implementation of a real inclusive education. This work aims to evaluate the teachers'sense of efficacy to implement inclusive practices by adapting a scale, with 153 teachers from regular and special education. Results demonstrate good factorial structure and reliability of the scale. Significant differences regarding: personal variables, as gender, age or educational background; professional variables, as teaching experience and contractual status, training and personal experience with children with special needs, were also found. The results and their implications for teacher professional development and research in an inclusive school are discussed.

Keywords: Inclusion. Self-efficacy. Teachers. Instrument.

\section{La autoeficacia de los docentes para implementar prácticas inclusivas: aportes para una reflexión sobre la inclusión educativa}

\section{Resumen}

En los últimos años, mucho ha cambiado en la legislación y en las prácticas para una escuela inclusiva, pasando por la preparación de las escuelas y el desarrollo profesional de los docentes. Sin embargo, es menos visible la investigación centrada sobre las prácticas inclusivas y el sentido de preparación de los docentes para una verdadera educación inclusiva. Con este trabajo se pretende evaluar la percepción de competencia de los docentes en la implementación de prácticas inclusivas mediante la adaptación de una escala, con 153 docentes de educación regular y educación especial. Los resultados permitieron encontrar una estructura factorial adecuada y buena fidelidad. Se encontraron también diferencias en función de: variables personales, como el género, la edad o el nivel educativo; variables profesionales, como tiempo de servicio, situación contractual, formación y experiencia personal con los niños con necesidades especiales. Se discuten los resultados y sus implicaciones para el desarrollo profesional docente y la investigación en el campo de la educación inclusiva.

Palabras clave: Inclusión. Autoeficacia. Profesorado. Instrumento. 


\section{Referências}

AGUIAR, C. et al. Desenhos de investigação de sujeito único em educação especial. Análise Psicológica, v. 29, n. 1, p. 167-78, 2011. doi:10.14417/ap.46

AINSCOW, M. Educação para todos: torná-la uma realidade. In: AINSCOW, M.; PORTER, G.; WANG, M. (Eds). Caminhos para as escolas inclusivas. Lisboa: Instituto de Inovação Educacional, 1997. p. 13-29.

. Necessidades especiais na sala de aula: um guia para a formação de professores. Lisboa: Instituto de Inovação Educacional, 1998.

ALI, M. M.; MUSTAPHA, R.; JELAS, Z. M. An empirical study on teachers' perceptions towards inclusive education in Malaysia. International Journal of Special Education, v. 21, n. 3, p. 36-44, Jan. 2006.

BANDURA, A. Self-efficacy: the exercise of control. New York: Freeman, 1997.

BEN-YEHUDA, S.; LEYSER, Y.; LAST, U. Teacher educational beliefs and sociometric status of Special Educational Needs (SEN) Students in inclusive classrooms. International Journal of Inclusive Education, v. 14, n. 1, p. 17-34, Feb. 2010.

CAPUTO, A.; LANGHER, V. Validation of the collaboration and support for inclusive teaching scale in special education teachers. Journal of Psychoeducational Assessment, v. 33, p. 210-22, May 2015. doi:10.1177/0734282914548335

CONDERMAN, G. Middle school co-teaching: effective practices and student reflections. Middle School Journal, v. 42, n.4, p. 24-31, 2011. doi: $10.2307 / 23047713$

COOK, B. G., et al. Bringing research to bear on practice: effecting evidence-based instruction for students with emotional or behavioral disorders. Education and Treatment of Children, v. 26, n. 4, p. 345-361, Nov. 2003.

COOK, B. G.; SCHIRMER, B. R. What is special about special education? Overview and analysis. Journal of Special Education, v. 37, n. 3, p. 200-5, Oct. 2003.

COOK, B. G.; TANKERSLEY, M.; LANDRUM, T. L. Determining evidence-based practices in special education. Exceptional Children, v. 75, n. 3, p. 365-83, Sep. 2009. 
CORREIA, L. M. Inclusão e necessidades educativas especiais: um guia para educadores e professores. Porto: Porto Editora, 2005.

. Inclusão e necessidades educativas especiais: um guia para educadores e professores. 2. ed. Porto: Porto Editora, 2008.

. O sistema educativo português e as necessidades educativas especiais ou quando inclusão quer dizer exclusão. In: CORREIA, L. M. (Org.). Educação especial e inclusão: quem disser que uma sobrevive sem a outra não está no seu perfeito juízo. Porto: Porto Editora, 2003. p. 11-39.

CURCIC, S. Inclusion in PK-12: an international perspective. International Journal of Inclusive Education, v. 13, n. 5, p. 517-38, Aug. 2009.

DIAS, P. C. et al. A experiência, atitude e sentido de eficácia do professor de educação especial em Portugal. In: RODRIGUES, A.; CASAL, J.; DIAS, P. C. (Orgs.). Educação especial: por uma escola de, com e para todos. Mangualde: Pedago, 2013. p. 177-90.

DIAS, P. C.; LEAL, A. R.; DIÁZ, J. Percepciones de los maestros de la ensañaza sobre la inclusión: datos preliminares. In: RAMIRO-SÁNCHEZ, T.; RAMIRO, M. T.; BERMÚDEZ, M. P. (Coords.). Avances en ciencias de la educación y del desarrollo. Granada: Asociación Española de Psicología Conductual, 2013. p. 397-401.

FREITAS, E. et al. Percepção dos pais em relação à inclusão de crianças com necessidades educativas especiais no ensino regular. Revista Educação Especial, v. 28, n. 52, 443-57, mayo-ago. 2015.

GIANGRECO, M. F.; BAUMGART, D. M.; DOYLE, M. B. How inclusion can facilitate teaching and learning. Intervention in School and Clinic, v. 30, n. 5, p.273-8, May 1995. doi:10.1177/105345129503000504

GREGUOL, M.; GOBBI, E.; CARRARO, A. Formação de professores para a educação especial: uma discussão sobre os modelos brasileiro e italiano. Revista Brasileira de Educação Especial, v. 19, n. 3, p. 307-24, jul.-set. 2013. doi:10.1590/S1413-65382013000300002

HARVEY, M. W. et al. Preservice teacher preparation for inclusion. An exploration of higher education teacher-training institutions. Remedial and Special Education, v. 31, n. 1, p. 24-33, Jan. 2010. 
HEIMAN, T. Teachers coping with changes: Including students with disabilities in mainstream classes: an international view. International Journal of Special Education, v.19, n.2, p. 91-103, 2004.

HUANG, C. Gender differences in academic self-efficacy: a meta-analysis. European Journal of Psychology of Education, v. 28, n. 1, p. 1-35, Jan. 2013. doi:10.1007/s10212-011-0097-y

KIVIET, A. M; MJI, A. Sex differences in self-efficacy beliefs of elementary science teachers. Psychological Reports, v. 92, n. 1, p. 333-8, Feb. 2003. doi:10.2466/pr0.2003.92.1.333

LIMA, J. A. As culturas colaborativas nas escolas: estruturas, processos e conteúdos. Porto: Porto Editora, 2002.

MALINEN, O.-P. et al. Exploring teacher self-efficacy for inclusive practices in three diverse countries. Teaching and Teacher Education, v. 33, p. 34-44, July 2013. doi:10.1016/j.tate.2013.02.004

MORGADO, J. Qualidade, inclusão e diferenciação. Lisboa: ISPA, 2003.

PINTO, I. P. A Inclusão como processo de socialização, equidade e aprendizagem. In: RODRIGUES, A.; CASAL, J.; DIAS, P. (Orgs.). Educação especial: por uma escola de, com e para todos. Mangualde: Pedago, 2013. p. 9-20.

PORTUGAL. Conselho Nacional de Educação. Lei n. ${ }^{\circ}$ 46/86. Lei de Bases do Sistema Educativo. 14 Out. 1986.

. Decreto-Lei n. ${ }^{\circ} 3 / 2008$, de 7 de Janeiro de 2008. No

desenvolvimento do regime jurídico estabelecido pela Lei n. ${ }^{\circ}$ 46/86, de 14 de Outubro, define os apoios especializados a prestar na educação pré-escolar e nos ensinos básico e secundário dos sectores público, particular e cooperativo. Diário da República. S.1, n. 4, p. 154-64, Jan. 2008

. Decreto-Lei n. ${ }^{\circ}$ 319/91, de 23 de Agosto de 1991. Aprova o regime de apoio a alunos com necessidades educativas especiais que frequentem estabelecimentos dos ensinos básico e secundário. Diário da República, S. 1.

RIGGS, I. M. Gender differences in elementary science teacher self-efficacy. In: ANNUAL MEETING OF THE AMERICAN EDUCATIONAL RESEARCH ASSOCIATION, 1991, Chicago, Ill. Chicago: American Educational Research Association, 1991. 
RODRIGUES, D.; NOGUEIRA, J. Educação especial e inclusiva em Portugal: fatos e opções. Revista Brasileira de Educação Especial, v. 17, n. 1, p. 3-20, jan./abr. 2011. doi:10.1590/S1413-65382011000100002

ROLDÃO, M. Colaborar é preciso: questões de qualidade e eficácia no trabalho dos professores. Noesis, n. 71, 24-29, out./dez. 2007.

SCHUNK, D. H.; MEECE, J. L. Self-efficacy development in adolescences. In URDAN, T.; PAJARES, F. (Eds.). Self-efficacy beliefs of adolescents. Greenwich: Information Age, 2005. p. 71-96.

SHARMA, U.; LOREMAN, T.; FORLIN, C. Measuring teacher efficacy to implement inclusive practices. Journal of Research in Special Educational Needs, v. 12, n. 1, p. 12-21, Jan. 2012. doi:10.1111/j.1471-3802.2011.01200.x

SILEO, J. M. Co-teaching: getting to know your partner. Teaching Exceptional Children, v. 43, n. 5, p. 32-8, 2011. doi:10.1177/004005991104300503

SPENCER, T. D.; DETRICH, R.; SLOCUM, T. A. Evidence-based practice: a framework for making effective decisions. Education and Treatment of Children, v. 35, n. 2, p. 127-51, 2012.

TSCHANNEN-MORAN, M.; WOOLFOLK HOY, A. Teacher efficacy: capturing and elusive construct. Teaching and Teacher Education, v. 17, p. 783-805, 2001.

TSCHANNEN-MORAN, M.; WOOLFOLK HOY, A.; HOY, W. K. Teacher efficacy: its meaning and measure. Review of Educational Research, v. 68, n.2, p. 202-48, 1998. doi:10.3102/00346543068002202

VILLA, R. A.; THOUSAND, J. S.; NEVIN, A. I. A guide to co-teaching: practial tips for facilitating student learning. Thousand Oaks: Corwin, 2008.

WORLD CONFERENCE ON SPECIAL NEEDS EDUCATION: Access and quality; 1994; Salamanca, Spain. Final report... Paris: Unesco, 1994.

ZIMMERMAN, B. J.; CLEARY, T. J. Adolescents' development of personal agency: the role of self-efficacy beliefs and self-regulatory skill. In: URDAN, T. PAJARES, F. (Eds.). Self-efficacy beliefs of adolescents. Greenwich: Information Age, 2006. p. 45-69. 
(c) (i) (:)

\section{Informações do autor}

Paulo C. Dias: Doutor. Professor da Faculdade de Filosofia e Ciências Sociais da Universidade Católica Portuguesa - UCP, Braga, Portugal. Contato: pcdias@braga.ucp.pt 